Pacific

Journal of

Mathematics

REPRESENTATIONS OF MULTILOOP ALGEBRAS

Michael LAU

Volume $245 \quad$ No. 1

March 2010 


\title{
REPRESENTATIONS OF MULTILOOP ALGEBRAS
}

\author{
MichAEL LAU
}

\begin{abstract}
We describe the finite-dimensional simple modules of all the (twisted and untwisted) multiloop algebras and classify them up to isomorphism.
\end{abstract}

\section{Introduction}

Multiloop algebras are multivariable generalizations of the loop algebras appearing in affine Kac-Moody theory. The study of these algebras and their extensions includes a substantial literature on (twisted and untwisted) multiloop, toroidal, and extended affine Lie algebras. This paper describes the finite-dimensional simple modules of multiloop algebras and classifies them up to isomorphism.

Let $\mathfrak{g}$ be a finite-dimensional simple Lie algebra over an algebraically closed field $F$ of characteristic zero. Suppose that $\sigma_{1}, \ldots, \sigma_{N}: \mathfrak{g} \rightarrow \mathfrak{g}$ are commuting automorphisms of finite orders $m_{1}, \ldots, m_{N}$, respectively. For each $i$, fix a primitive $m_{i}$-th root of unity $\xi_{i} \in F$. Then $\mathfrak{g}$ decomposes into common eigenspaces relative to these automorphisms:

$$
\mathfrak{g}=\bigoplus_{\bar{k} \in G} \mathfrak{g}_{\bar{k}}
$$

where $\mathfrak{g}_{\bar{k}}=\left\{x \in \mathfrak{g} \mid \sigma_{i} x=\xi_{i}^{k_{i}} x\right\}$ and $\bar{k}$ is the image of each $k \in \mathbb{Z}^{N}$ under the canonical map $\mathbb{Z}^{N} \rightarrow G=\mathbb{Z} / m_{1} \mathbb{Z} \times \cdots \times \mathbb{Z} / m_{N} \mathbb{Z}$. The multiloop algebra of $\mathfrak{g}$, relative to these automorphisms, is the Lie algebra

$$
\mathscr{L}=\mathscr{L}\left(\mathfrak{g} ; \sigma_{1}, \ldots, \sigma_{N}\right)=\bigoplus_{k \in \mathbb{Z}^{N}} \mathfrak{g}_{\bar{k}} \otimes F t^{k},
$$

where $F t^{k}$ is the span of $t^{k}=t_{1}^{k_{1}} \cdots t_{N}^{k_{N}}$, and multiplication is defined pointwise. If the automorphisms $\sigma_{1}, \ldots, \sigma_{N}$ are all trivial, $\mathscr{L}$ is called an untwisted multiloop algebra. Otherwise, it is a twisted multiloop algebra.

In the one variable case (untwisted and twisted loop algebras), a proof of the classification of the finite-dimensional simple modules appears in [Chari 1986;

MSC2000: 17B10, 17B65.

Keywords: multiloop algebras, toroidal Lie algebras, finite-dimensional modules.

The author gratefully acknowledges support from the University of Windsor and the Natural Sciences and Engineering Research Council of Canada. 
Chari and Pressley 1986; 1987; 1988]. A complete list of these modules also appears explicitly in [Rao 1993], and a very recent paper [Chari et al. 2008] gives a detailed discussion of this problem in the twisted loop case.

A description of the finite-dimensional simple modules of the untwisted multiloop Lie algebras was first given by S. E. Rao [2001]. Subsequent work by P. Batra [2004] provided a complete (but redundant) list of the finite-dimensional simple modules when $\sigma_{1}$ is a diagram automorphism and the other automorphisms $\sigma_{2}, \ldots, \sigma_{N}$ are all trivial.

In the one variable case, every twisted loop algebra $\mathscr{L}(\mathfrak{g} ; \tau \circ \gamma)$ defined by an inner automorphism $\tau$ and a diagram automorphism $\gamma$ is isomorphic to $\mathscr{L}(\mathfrak{g} ; \gamma)$ [Kac 1990, Proposition 8.5]. It thus suffices to consider twists only by diagram automorphisms in this setting. Unfortunately, this is far from true in the multivariable case. See [Gille and Pianzola 2007, Remark 5.9], for instance. It has recently been shown that the centreless core of almost every extended affine Lie algebra is a multiloop algebra [Allison et al. 2009], using results of [Allison et al. 2008; Neher 2004]. Even for these multiloop algebras, any number of the automorphisms $\sigma_{i}$ can be nontrivial, and any number of them can fail to be diagram automorphisms.

In this paper, we consider an arbitrary (twisted or untwisted) multiloop algebra $\mathscr{L}$. From any ideal $\mathscr{I}$ of $\mathscr{L}$, we construct a $G$-graded ideal $I=I(\mathscr{I})$ of the ring $R=F\left[t_{1}^{ \pm 1}, \ldots, t_{N}^{ \pm 1}\right]$ of Laurent polynomials. If $\mathscr{I}$ is the kernel of a finitedimensional irreducible representation, the $\overline{0}$-component $I_{\overline{0}}$ of $I$ turns out to be a radical ideal of the $\overline{0}$-component of $R$. The resulting decomposition of $I_{\overline{0}}$ into an intersection of a finite number of maximal ideals produces an isomorphism

$$
\psi_{a}: \mathscr{L} / \mathscr{I} \rightarrow \mathfrak{g} \oplus \cdots \oplus \mathfrak{g} \quad(r \text { copies })
$$

whose composition with the quotient map $\pi: \mathscr{L} \rightarrow \mathscr{L} / \mathscr{I}$ is evaluation at an $r$-tuple $a=\left(a_{1}, \ldots, a_{r}\right)$ of points $a_{i} \in\left(F^{\times}\right)^{N}$ :

$$
\psi_{a} \circ \pi: x \otimes f(t) \mapsto\left(f\left(a_{1}\right) x, \ldots, f\left(a_{r}\right) x\right)
$$

for any $x \otimes f(t) \in \mathscr{L}$. Since the finite-dimensional simple modules of the semisimple Lie algebra $\mathfrak{g} \oplus \cdots \oplus \mathfrak{g}$ are the tensor products of finite-dimensional simple modules for $\mathfrak{g}$, we obtain a complete (but redundant) list of the finite-dimensional irreducible representations of $\mathscr{L}$ (Corollary 4.4). Namely, any finite-dimensional simple module for $\mathscr{L}$ is of the form

$$
V(\lambda, a)=V_{\lambda_{1}}\left(a_{1}\right) \otimes \cdots \otimes V_{\lambda_{r}}\left(a_{r}\right),
$$

where $V_{\lambda_{i}}$ is the $\mathfrak{g}$-module of dominant integral highest weight $\lambda_{i}$, and $V_{\lambda_{i}}\left(a_{i}\right)$ is the $\mathscr{L}$-module obtained by evaluating elements of $\mathscr{L}$ at the point $a_{i}$, and then letting the resulting element of $\mathfrak{g}$ act on $V_{\lambda_{i}}$. The $r$-tuples $a=\left(a_{1}, \ldots, a_{r}\right)$ that occur in this process must satisfy the condition that the points $m\left(a_{i}\right)$ are all distinct, 
where $m\left(a_{i}\right)=\left(a_{i 1}^{m_{1}}, \ldots, a_{i N}^{m_{N}}\right)$ is determined by the orders $m_{1}, \ldots, m_{N}$ of the automorphisms $\sigma_{1}, \ldots, \sigma_{N}$. Conversely, the $\mathscr{L}$-module $V(\lambda, a)$ is finite-dimensional and simple if the $a_{i}$ satisfy this condition (Theorem 4.5).

In the second half of the paper, we establish necessary and sufficient conditions for $\mathscr{L}$-modules $V(\lambda, a)$ and $V(\mu, b)$ to be isomorphic. Namely, we "pull back" a triangular decomposition $N_{-} \oplus H \oplus N_{+}$of $\mathfrak{g} \oplus \cdots \oplus \mathfrak{g}$ to a triangular decomposition $\psi_{a}^{-1}\left(N_{-}\right) \oplus \psi_{a}^{-1}(H) \oplus \psi_{a}^{-1}\left(N_{+}\right)$of $\mathscr{L} / \mathscr{I}$. The modules $V(\lambda, a)$ and $V(\mu, b)$ are highest weight with respect to this decomposition of $\mathscr{L} / \mathscr{I}$, and they are isomorphic if and only if they have the same highest weights. We conclude with three equivalent criteria for isomorphism in terms of an explicit formula in Theorem 5.4, orbits under a group action in Corollary 5.9, and equivariant maps in Corollary 5.10. These are the first such isomorphism results for modules in any multiloop setting.

Interestingly, the triangular decomposition $N_{-} \oplus H \oplus N_{+}$is replaced with a new triangular decomposition $\psi_{b} \psi_{a}^{-1}\left(N_{-}\right) \oplus \psi_{b} \psi_{a}^{-1}(H) \oplus \psi_{b} \psi_{a}^{-1}\left(N_{+}\right)$of $\mathfrak{g}^{\oplus r}$ in the computation of the highest weight of $V(\mu, b)$. Unlike diagram automorphisms, arbitrary finite-order automorphisms $\sigma_{i}$ often fail to stabilize any triangular decomposition of a finite-dimensional semisimple Lie algebra. This fact is reflected in the change of triangular decomposition on $\mathfrak{g}^{\oplus r}$, and it is one of the reasons that past work considered only twists by diagram automorphisms.

Another novelty in this classification is the passage from twists by a single nontrivial automorphism $\sigma_{1}$ to a family of nontrivial automorphisms $\sigma_{1}, \ldots, \sigma_{N}$. Here the major obstacle to past approaches was reliance on the representation theory of the fixed point subalgebra $\mathfrak{g}_{\overline{0}}$ under the action of the automorphisms. While this was a great success when working with twists by a single automorphism, it cannot be used when considering twists by more than one automorphism, since the algebra $\mathfrak{g}_{\overline{0}}$ is then often 0 . We avoid this pitfall by using a new approach that does not rely on the usual Dynkin diagram folding arguments.

We expect the methods of this paper to be widely applicable. For example, the arguments given here classify the finite-dimensional simple modules of the Lie algebra $\operatorname{Map}(X, \mathfrak{g})$ of $\mathfrak{g}$-valued regular functions on any affine variety $X$; namely, they are tensor products of evaluation modules at distinct points of $X$. Since the release of earlier versions of this paper, our approach has already been adapted to classify modules for Lie algebras $\mathfrak{g} \otimes A$ of $\mathfrak{g}$-valued functions on affine schemes $\operatorname{Spec}(A)$ and their invariants under more general finite group actions [Chari et al. 2009; Neher et al. 2009]. Another promising direction is the classification of $\mathbb{Z}^{N}$-graded-simple modules of $L\left(\mathfrak{g} ; \sigma_{1}, \ldots, \sigma_{N}\right)$ with finite-dimensional graded components. See [Pal and Batra 2008; Rao 2001] for partial results.

Notation. Throughout this paper, $F$ will be an algebraically closed field of characteristic zero. All Lie algebras, linear spans, and tensor products will be taken 
over $F$ unless otherwise indicated. We will denote the integers by $\mathbb{Z}$, the nonnegative integers by $\mathbb{Z}_{+}$, and the nonzero elements of $F$ by $F^{\times}$.

\section{Multiloop algebras and their ideals}

The following proposition is an immediate consequence of general facts about reductive Lie algebras.

Proposition 2.1. Let $L$ be a perfect Lie algebra over $F$, and let $\phi: L \rightarrow$ End $V$ be a finite-dimensional irreducible representation. Then $L / \operatorname{ker} \phi$ is a semisimple Lie algebra.

Proof. The representation $\phi$ descends to a faithful representation of $L / \operatorname{ker} \phi$. By [Bourbaki 1960, Proposition 6.4.5], any Lie algebra with a faithful finitedimensional irreducible representation is reductive. Also, $L$ is perfect. Therefore, $L / \operatorname{ker} \phi$ is perfect and reductive, and hence semisimple.

We now focus our attention on multiloop algebras. Let $\mathfrak{g}$ be a finite-dimensional simple Lie algebra over $F$, and let $R=F\left[t_{1}^{ \pm 1}, \ldots, t_{N}^{ \pm 1}\right]$ be the commutative algebra of Laurent polynomials in $N$ variables. The untwisted multiloop algebra is the Lie algebra $\mathfrak{g} \otimes R$ with (bilinear) pointwise multiplication given by

$$
[x \otimes f, y \otimes g]=[x, y] \otimes f g \quad \text { for all } x, y \in \mathfrak{g} \text { and } f, g \in R .
$$

Suppose that $\mathfrak{g}$ is equipped with $N$ commuting automorphisms $\sigma_{1}, \ldots, \sigma_{N}: \mathfrak{g} \rightarrow \mathfrak{g}$ of finite orders $m_{1}, \ldots, m_{N}$, respectively. For each $i$, fix $\xi_{i} \in F$ to be a primitive $m_{i}$-th root of 1 . Then $\mathfrak{g}$ has a common eigenspace decomposition $\mathfrak{g}=\bigoplus_{\bar{k} \in G} \mathfrak{g}_{\bar{k}}$, where $\bar{k}$ is the image of $k=\left(k_{1}, \ldots, k_{N}\right) \in \mathbb{Z}^{N}$ under the canonical map

$$
\mathbb{Z}^{N} \rightarrow G=\mathbb{Z} / m_{1} \mathbb{Z} \times \cdots \times \mathbb{Z} / m_{N} \mathbb{Z},
$$

and

$$
\mathfrak{g}_{\bar{k}}=\left\{x \in \mathfrak{g} \mid \sigma_{i} x=\xi_{i}^{k_{i}} x \text { for } i=1, \ldots, N\right\} .
$$

The (twisted) multiloop algebra $\mathscr{L}=\mathscr{L}\left(\mathfrak{g} ; \sigma_{1}, \ldots, \sigma_{N}\right)$ is the Lie subalgebra

$$
\mathscr{L}=\bigoplus_{k \in \mathbb{Z}^{N}} \mathfrak{g}_{\bar{k}} \otimes F t^{k} \subseteq \mathfrak{g} \otimes R
$$

where $t^{k}=t_{1}^{k_{1}} \cdots t_{N}^{k_{N}}$ is multiindex notation.

Note that $R$ has a $G$-grading

$$
R=\bigoplus_{\bar{k} \in G} R_{\bar{k}}
$$


where $R_{\overline{0}}=F\left[t_{1}^{ \pm m_{1}}, \ldots, t_{N}^{ \pm m_{N}}\right]$ and $R_{\bar{k}}=t^{k} R_{\overline{0}}$ for every $k \in \mathbb{Z}^{N}$. In this notation,

Fix an $F$-basis

$$
\mathscr{L}=\bigoplus_{\bar{k} \in G}\left(\mathfrak{g}_{\bar{k}} \otimes R_{\bar{k}}\right)
$$

$$
\left\{x_{\bar{k} j} \mid j=1, \ldots, \operatorname{dim} \mathfrak{g}_{\bar{k}}\right\}
$$

of $\mathfrak{g}_{\bar{k}}$ for all $\bar{k} \in G$. Then

$$
\mathscr{L}=\bigoplus_{\bar{k} \in G} \bigoplus_{j=1}^{\operatorname{dim} \mathfrak{g}_{\bar{k}}}\left(F x_{\bar{k} j} \otimes R_{\bar{k}}\right) .
$$

Since $\mathfrak{g}$ is simple (hence perfect) and graded, each $x_{\bar{k} j}$ can be expressed as a sum of brackets of homogeneous elements $y, z \in \mathfrak{g}$, with $\operatorname{deg} y+\operatorname{deg} z=\bar{k}$. For each such $k \in \mathbb{Z}^{N}$ and pair $y, z$, there exist $a, b \in \mathbb{Z}^{N}$ with $\operatorname{deg} y=\bar{a}, \operatorname{deg} z=\bar{b}$, and $a+b=k$. Then the sum of the brackets $\left[y \otimes t^{a}, z \otimes t^{b}\right]$ will be $x_{\bar{k} j} \otimes t^{k}$. Since these elements span $\mathscr{L}$, it is clear that $\mathscr{L}$ is perfect. See also [Allison et al. 2006, Lemma 4.9].

Let $\pi_{\bar{k} j}$ be the projection $\pi_{\bar{k} j}: \mathscr{L} \rightarrow F x_{\bar{k} j} \otimes R_{\bar{k}}$ relative to the decomposition (2.4). We will view $\pi_{\bar{k} j}$ as a projection $\mathscr{L} \rightarrow R_{\bar{k}}$ by identifying $x_{\bar{k} j} \otimes f$ with $f$ for all $f \in R_{\bar{k}}$. Let $\mathscr{I}$ be an ideal of the Lie algebra $\mathscr{L}$, and let $I=I(\mathscr{F})$ be the ideal of $R$ generated by

$$
\bigcup_{\bar{k} \in G} \bigcup_{j=1}^{\operatorname{dim} \mathfrak{g}_{\bar{k}}} \pi_{\bar{k} j}(\mathscr{Y}) .
$$

Note that the definition of $I$ is independent of the choice of homogeneous basis $\left\{x_{\bar{k} j}\right\}$ of $\mathfrak{g}$, and the ideal $I$ is $G$-graded since its generators are homogeneous with respect to the $G$-grading of $R$. That is,

$$
I=\bigoplus_{\bar{k} \in G} I_{\bar{k}}, \quad \text { where } I_{\bar{k}}=I \cap R_{\bar{k}}
$$

Moreover, $t^{\ell-k} I_{\bar{k}} \subseteq I \cap R_{\bar{\ell}}=I_{\bar{\ell}}=t^{\ell-k}\left(t^{k-\ell} I_{\bar{\ell}}\right) \subseteq t^{\ell-k} I_{\bar{k}}$, so

$$
I_{\bar{\ell}}=t^{\ell-k} I_{\bar{k}} \text { for all } k, \ell \in \mathbb{Z}^{N} .
$$

We will use the following technical lemma to show that $\mathscr{I}=\mathscr{L} \cap(\mathfrak{g} \otimes I)$.

Lemma 2.6. Let

$$
Y=\sum_{\bar{r} \in G} \sum_{n=1}^{\operatorname{dim} \mathfrak{g}_{\bar{r}}} x_{\bar{r} n} \otimes \pi_{\bar{r} n}(Y) \in \mathscr{I} .
$$

Then $x_{\bar{k} i} \otimes t^{k-\ell} \pi_{\bar{\ell} j}(Y) \in \mathscr{I}$ for all $k, \ell \in \mathbb{Z}^{N}, 1 \leq i \leq \operatorname{dim} \mathfrak{g}_{\bar{k}}$, and $1 \leq j \leq \operatorname{dim} \mathfrak{g}_{\bar{\ell}}$. 
Proof. The finite-dimensional simple Lie algebra $\mathfrak{g}$ is a finite-dimensional simple $\mathfrak{g}$-module (and hence a finite-dimensional simple $\mathfrak{U}(\mathfrak{g})$-module) under the adjoint action of $\mathfrak{g}$. Fix $k, \ell \in \mathbb{Z}^{N}, i \in\left\{1, \ldots, \operatorname{dim} \mathfrak{g}_{\bar{k}}\right\}$ and $j \in\left\{1, \ldots, \operatorname{dim} \mathfrak{g}_{\bar{\ell}}\right\}$. By the Jacobson density theorem, there exists $u \in \mathfrak{U}(\mathfrak{g})$ such that

$$
u \cdot x_{\bar{r} n}= \begin{cases}x_{\bar{k} i} & \text { if } \bar{r}=\bar{\ell} \text { and } n=j \\ 0 & \text { otherwise }\end{cases}
$$

for all $\bar{r} \in G$ and $n \in\left\{1, \ldots, \mathfrak{g}_{\bar{r}}\right\}$. By the Poincaré-Birkhoff-Witt theorem, we can write $u=\sum_{s=1}^{a} p_{s}$, where $a \geq 1$ and each $p_{s}$ is a monomial in the variables in $\left\{x_{\bar{r} n} \mid \bar{r} \in G, n=1, \ldots, \operatorname{dim} \mathfrak{g}_{\bar{r}}\right\}$. Considering the induced $G$-grading of $\mathfrak{U}(\mathfrak{g})$, we can assume that each $p_{s}$ is homogeneous of degree $\overline{k-\ell}$. Write

$$
p_{s}=c_{s} \prod_{\bar{r} \in G} \prod_{n=1}^{\operatorname{dim} \mathfrak{g}_{\bar{r}}}\left(x_{\bar{r} n}\right)^{b_{\bar{r} n}^{(s)}}, \quad \text { where } c_{s} \in F \text { and } b_{\bar{r} n}^{(s)} \in \mathbb{Z}_{+} \text {. }
$$

Since $p_{s}$ is homogeneous of degree $\overline{k-\ell}$ in the $G$-grading of $\mathfrak{U}(\mathfrak{g})$, we can choose $\alpha(s, \bar{r}, n, 1), \alpha(s, \bar{r}, n, 2), \ldots, \alpha\left(s, \bar{r}, n, b_{\bar{r} n}^{(s)}\right) \in \mathbb{Z}^{N}$ for each $s \in\{1, \ldots, a\}$, $\bar{r} \in G$, and $n \in\left\{1, \ldots, \operatorname{dim} \mathfrak{g}_{\bar{r}}\right\}$ so that

(i) $\bar{r}=\overline{\alpha(s, \bar{r}, n, 1)}=\cdots=\overline{\alpha\left(s, \bar{r}, n, b_{\bar{r} n}^{(s)}\right)}$ and

(ii) $\sum_{\bar{r} \in G} \sum_{n=1}^{\operatorname{dim} \mathfrak{g}_{\bar{r}}} \sum_{b=1}^{b_{\bar{r} n}^{(s)}} \alpha(s, \bar{r}, n, b)=k-\ell$.

Then

$$
\widetilde{p}_{s}=c_{s} \prod_{\bar{r} \in G} \prod_{n=1}^{\operatorname{dim} \mathfrak{g}_{\bar{r}}} \prod_{b=1}^{b_{\bar{r} n}^{(s)}}\left(x_{\bar{r} n} \otimes t^{\alpha(s, \bar{r}, n, b)}\right)
$$

is in the universal enveloping algebra $\mathfrak{U}(\mathscr{L})$ of $\mathscr{L}$, which acts on $\mathscr{I}$ via the adjoint action of $\mathscr{L}$ on $\mathscr{I}$, and $\sum_{s=1}^{a} \widetilde{p}_{s} . Y=x_{\bar{k} i} \otimes t^{k-\ell} \pi_{\bar{\ell} j}(Y)$, so $x_{\bar{k} i} \otimes t^{k-\ell} \pi_{\bar{\ell} j}(Y) \in \mathscr{I}$.

Proposition 2.7. In the notation introduced above,

$$
\begin{aligned}
\mathscr{I} & =\mathscr{L} \cap(\mathfrak{g} \otimes I) \\
& =\bigoplus_{\bar{k} \in G} \mathfrak{g}_{\bar{k}} \otimes I_{\bar{k}} .
\end{aligned}
$$

Proof. The second equality (2.9) and the inclusion $\mathscr{I} \subseteq \mathscr{L} \cap(\mathfrak{g} \otimes I)$ are clear, so it remains only to verify the reverse inclusion $\mathscr{L} \cap(\mathfrak{g} \otimes I) \subseteq \mathscr{I}$. In light of (2.9), it suffices to show that $x_{\bar{k} i} \otimes f \in \mathscr{I}$ for all $\bar{k} \in G, i \in\left\{1, \ldots, \operatorname{dim} \mathfrak{g}_{\bar{k}}\right\}$, and $f \in I_{\bar{k}}$. 
By the definition of $I$, there exist $Y_{\bar{\ell} j} \in \mathscr{I}$ and $f_{\bar{\ell} j} \in R_{\overline{k-\ell}}$ such that

$$
f=\sum_{\bar{\ell} \in G} \sum_{j=1}^{\operatorname{dim} \mathfrak{g}_{\bar{\ell}}} f_{\bar{\ell} j} \pi_{\bar{\ell} j}\left(Y_{\bar{\ell} j}\right) .
$$

By Lemma 2.6, $x_{\bar{k} i} \otimes t^{r} \pi_{\bar{\ell} j}\left(Y_{\bar{\ell} j}\right) \in \mathscr{I}$ for all $r, \ell \in \mathbb{Z}^{N}$ satisfying $\bar{r}=\overline{k-\ell}$. Since each $f_{\bar{\ell} j} \in R_{\overline{k-\ell}}$ is an $F$-linear combination of $\left\{t^{r} \mid \bar{r}=\overline{k-\ell}\right\}$, we see that

$$
x_{\bar{k} i} \otimes f_{\bar{\ell} j} \pi_{\bar{\ell} j}\left(Y_{\bar{\ell} j}\right) \in \mathscr{I}
$$

for all $\bar{\ell} \in G$ and $j=1, \ldots, \operatorname{dim} \mathfrak{g}_{\bar{\ell}}$. Thus $x_{\bar{k} i} \otimes f \in \mathscr{I}$.

We close this section by considering the structure of $I_{\overline{0}} \subseteq R_{\overline{0}}$ in the case where $\mathscr{I}$ is the kernel of an irreducible finite-dimensional representation of $\mathscr{L}$. Clearly $I_{\overline{0}}$ is an ideal of $R_{\overline{0}}$. Moreover, it is a radical ideal:

Proposition 2.10. Let $\phi: \mathscr{L} \rightarrow$ End $V$ be a finite-dimensional irreducible representation of the multiloop algebra $\mathscr{L}$, and let $\mathscr{I}=\operatorname{ker} \phi$. Define $I=I(\mathscr{I}) \subseteq R$ as above. Then the graded component $I_{\overline{0}}$ is a radical ideal of $R_{\overline{0}}$.

Proof. Suppose $p$ is an element of $\sqrt{I_{\overline{0}}}$, the radical of the ideal $I_{\overline{0}}=I \cap R_{\overline{0}}$ of $R_{\overline{0}}$. Choose $k \in \mathbb{Z}^{N}$ so that $\mathfrak{g}_{\bar{k}} \neq 0$, and let $x \in \mathfrak{g}_{\bar{k}}$ be a nonzero element.

For $y \otimes f \in \mathscr{L}$, let $\langle y \otimes f\rangle \subseteq \mathscr{L}$ be the ideal (of $\mathscr{L}$ ) generated by $y \otimes f$. Let $J=\left\langle x \otimes t^{k} p\right\rangle$, and note that the $n$-th term $J^{(n)}$ in the derived series of $J$ satisfies $J^{(n)} \subseteq \mathscr{L} \cap\left(\mathfrak{g} \otimes\left\langle p^{n}\right\rangle\right)$, where $\left\langle p^{n}\right\rangle$ is the principal ideal of $R$ generated by $p^{n}$. Since $I_{\bar{\ell}}=t^{\ell} I_{\overline{0}}$ for all $\ell \in \mathbb{Z}^{N}$ by (2.5), and since $p^{n} \in I_{\overline{0}}$ for $n$ sufficiently large, we see that $J^{(n)} \subseteq \mathscr{L} \cap(\mathfrak{g} \otimes I)$ for $n \gg 0$. Then by Proposition 2.7, $J^{(n)} \subseteq \mathscr{I}$, so

$$
\frac{J+\mathscr{I}}{\mathscr{I}} \subseteq \operatorname{Rad}(\mathscr{L} / \mathscr{\Phi})
$$

Since $\operatorname{Rad}(\mathscr{L} / \mathscr{I})=0$ by Proposition 2.1 , we see that $x \otimes t^{k} p \in \mathscr{I}$. That is, $p=t^{-k}\left(t^{k} p\right) \in t^{-k} I_{\bar{k}}=I_{\overline{0}}$, and thus $\sqrt{I_{\overline{0}}}=I_{\overline{0}}$.

\section{Some commutative algebra}

In this short section, we recall some basic commutative algebra that will be useful for classifying modules for multiloop algebras. Let $F, F^{\times}$, and $R$ be as before. For any ideal $I \subseteq R$, let $\mathscr{V}(I)=\left\{x \in\left(F^{\times}\right)^{N} \mid f(x)=0\right.$ for all $\left.f \in I\right\}$ be the (quasiaffine) variety corresponding to $I$, and let $\operatorname{Poly}(S)=\{g \in R \mid g(s)=0$ for all $s \in S\}$ be the ideal associated with any subset $S \subseteq\left(F^{\times}\right)^{N}$.

Proposition 3.1. Let $I$ be an ideal of $R=F\left[t_{1}^{ \pm 1}, \ldots, t_{N}^{ \pm 1}\right]$. Then

$$
\operatorname{Poly}(\mathscr{V}(I))=\sqrt{I}
$$


Proof. It is straightforward to verify that the usual proofs of the Hilbert Nullstellensatz (see [Atiyah and Macdonald 1969, page 85] for instance) also hold for this Laurent polynomial analogue.

The following crucial lemma is an easy consequence of Proposition 3.1:

Lemma 3.2. Let $J$ be a radical ideal of $R$ for which the quotient $R / J$ is a finitedimensional vector space over $F$. Then there exist distinct points $a_{1}, \ldots, a_{r} \in$ $\left(F^{\times}\right)^{N}$ such that

$$
J=\mathfrak{m}_{a_{1}} \cap \cdots \cap \mathfrak{m}_{a_{r}},
$$

where $\mathfrak{m}_{a_{i}}=\left\langle t_{1}-a_{i 1}, \ldots, t_{N}-a_{i N}\right\rangle$ is the maximal ideal corresponding to $a_{i}=$ $\left(a_{i 1}, \ldots, a_{i N}\right)$ for $i=1, \ldots, r$. Moreover, the set $\left\{a_{1}, \ldots, a_{r}\right\}$ is unique.

Proof. Clearly, $a \in \mathscr{V}(J)$ implies that $J \subseteq \mathfrak{m}_{a}$, so $J \subseteq \bigcap_{a \in \mathscr{V}(J)} \mathfrak{m}_{a}$. Conversely, if $f \in \bigcap_{a \in \mathscr{V}(J)} \mathfrak{m}_{a}$ and $x \in \mathscr{V}(J)$, then $f(x)=0$ and $f \in \operatorname{Poly}(\mathscr{V}(J))=\sqrt{J}=J$. Hence $J=\bigcap_{a \in \mathscr{V}(J)} \mathfrak{m}_{a}$.

Since $J \subseteq \mathfrak{m}_{a_{1}} \cap \cdots \cap \mathfrak{m}_{a_{r}}$ for all subsets $\left\{a_{1}, \ldots, a_{r}\right\} \subseteq \mathscr{V}(J)$, we see that the ( $F$-vector space) dimension of $R /\left(\mathfrak{m}_{a_{1}} \cap \cdots \cap \mathfrak{m}_{a_{r}}\right)$ is bounded by $\operatorname{dim}_{F}(R / J)$. Take a finite collection $\left\{a_{1}, \ldots, a_{r}\right\}$ of points in $\mathscr{V}(J)$ for which this dimension is maximal. Then $\mathfrak{m}_{a_{1}} \cap \cdots \cap \mathfrak{m}_{a_{r}} \cap \mathfrak{m}_{a_{r+1}}=\mathfrak{m}_{a_{1}} \cap \cdots \cap \mathfrak{m}_{a_{r}}$ for all points $a_{r+1} \in \mathscr{V}(J)$, so

$$
J=\bigcap_{b \in \mathscr{V}(J)} \mathfrak{m}_{b}=\mathfrak{m}_{a_{1}} \cap \cdots \cap \mathfrak{m}_{a_{r}} \cap\left(\bigcap_{b \in \mathscr{V}(J)} \mathfrak{m}_{b}\right)=\mathfrak{m}_{a_{1}} \cap \cdots \cap \mathfrak{m}_{a_{r}}
$$

To see that $\left\{a_{1}, \ldots, a_{r}\right\} \subseteq\left(F^{\times}\right)^{N}$ is uniquely determined, suppose that $J=$ $\mathfrak{m}_{a_{1}} \cap \cdots \cap \mathfrak{m}_{a_{r}}=\mathfrak{m}_{b_{1}} \cap \cdots \cap \mathfrak{m}_{b_{s}}$ for some $a_{1}, \ldots, a_{r}, b_{1}, \ldots, b_{s} \in\left(F^{\times}\right)^{N}$. Then

$$
\begin{aligned}
\left\{a_{1}, \ldots, a_{r}\right\} & =\mathscr{V}\left(\mathfrak{m}_{a_{1}} \cap \cdots \cap \mathfrak{m}_{a_{r}}\right) \\
& =\mathscr{V}(J) \\
& =\mathscr{V}\left(\mathfrak{m}_{b_{1}} \cap \cdots \cap \mathfrak{m}_{b_{s}}\right)=\left\{b_{1}, \ldots, b_{s}\right\} .
\end{aligned}
$$

Note that the ideal $I_{\overline{0}} \subseteq R_{\overline{0}}$ of Proposition 2.10 is radical and cofinite. Viewing $R_{\overline{0}}=F\left[t_{1}^{ \pm m_{1}}, \ldots, t_{N}^{ \pm m_{N}}\right]$ as the ring of Laurent polynomials in the variables $t_{1}^{m_{1}}, \ldots, t_{N}^{m_{N}}$, we see that

$$
I_{\overline{0}}=M_{a_{1}} \cap \cdots \cap M_{a_{r}},
$$

where $\left\{a_{1}, \ldots, a_{r}\right\}=\mathscr{V}\left(I_{\overline{0}}\right)$ is a set of distinct points in $\left(F^{\times}\right)^{N}$, and

$$
M_{a_{i}}=\left\langle t_{1}^{m_{1}}-a_{i 1}, \ldots, t_{N}^{m_{N}}-a_{i N}\right\rangle_{R_{\overline{0}}}
$$

is the maximal ideal of $R_{\overline{0}}$ corresponding to the point $a_{i}=\left(a_{i 1}, \ldots, a_{i N}\right)$. Then by the Chinese remainder theorem, we have the following corollary: 
Corollary 3.3. Let $I_{\overline{0}}$ and $R_{\overline{0}}$ be as in Proposition 2.10. Then there exist unique (up to reordering) points $a_{1}, \ldots, a_{r} \in\left(F^{\times}\right)^{N}$ such that the canonical map

$$
R_{\overline{0}} / I_{\overline{0}} \rightarrow R_{\overline{0}} / M_{a_{1}} \times \cdots \times R_{\overline{0}} / M_{a_{r}}, \quad f+I_{\overline{0}} \mapsto\left(f+M_{a_{1}}, \ldots, f+M_{a_{r}}\right)
$$

is a well-defined F-algebra isomorphism.

\section{Classification of simple modules}

We now return to classifying the finite-dimensional simple modules of multiloop algebras. As in Section 2, let $\mathfrak{g}$ be a finite-dimensional simple Lie algebra, and let $\phi: \mathscr{L} \rightarrow$ End $V$ be a finite-dimensional irreducible representation of a multiloop algebra $\mathscr{L}=\mathscr{L}\left(\mathfrak{g} ; \sigma_{1}, \ldots, \sigma_{N}\right)$ defined by commuting automorphisms $\sigma_{1}, \ldots, \sigma_{N}$ of order $m_{1}, \ldots, m_{N}$, respectively.

Define $\mathscr{I}, I, G$, and $R$ as in Section 2. Then we see that

$$
\mathscr{L}=\bigoplus_{\bar{k} \in G} \mathfrak{g}_{\bar{k}} \otimes R_{\bar{k}} \quad \text { and } \quad \mathscr{I}=\bigoplus_{\bar{k} \in G} \mathfrak{g}_{\bar{k}} \otimes I_{\bar{k}},
$$

by Proposition 2.7. Since $\mathscr{I}$ is a $G$-graded ideal of $\mathscr{L}$, we have

$$
\mathscr{L} / \mathscr{I}=\bigoplus_{\bar{k} \in G}\left(\left(\mathfrak{g}_{\bar{k}} \otimes R_{\bar{k}}\right) /\left(\mathfrak{g}_{\bar{k}} \otimes I_{\bar{k}}\right)\right)=\bigoplus_{\bar{k} \in G} \mathfrak{g}_{\bar{k}} \otimes\left(R_{\bar{k}} / I_{\bar{k}}\right) .
$$

Each graded component $R_{\bar{k}} / I_{\bar{k}}$ of $R / I$ is an $R_{\overline{0}}$-module, and it is easy to check that the map

$$
\mu_{k}: R_{\overline{0}} / I_{\overline{0}} \rightarrow R_{\bar{k}} / I_{\bar{k}}, \quad f+I_{\overline{0}} \mapsto t^{k} f+I_{\bar{k}}
$$

is a well-defined $R_{\overline{0}}$-module homomorphism for each $k \in \mathbb{Z}^{N}$ and $f \in R_{\overline{0}}$. By (2.2) and (2.5), $R_{\bar{k}}=t^{k} R_{\overline{0}}$ and $t^{-k} I_{\bar{k}}=I_{\overline{0}}$, so the map $\mu_{k}$ is both surjective and injective. Hence the following lemma holds:

Lemma 4.1. Let $k \in \mathbb{Z}^{N}$. Then the map $\mu_{k}: R_{\overline{0}} / I_{\overline{0}} \rightarrow R_{\bar{k}} / I_{\bar{k}}$ is a well-defined isomorphism of $R_{\overline{0}}$-modules. In particular, each graded component $R_{\bar{k}} / I_{\bar{k}}$ has the same dimension (as a vector space), that is, $\operatorname{dim}\left(R_{\bar{k}} / I_{\bar{k}}\right)=\operatorname{dim}\left(R_{\overline{0}} / I_{\overline{0}}\right)$.

Let $a_{1}, \ldots, a_{r} \in\left(F^{\times}\right)^{N}$ be the (unique) points defined by Corollary 3.3, and let $b_{i}=\left(b_{i 1}, \ldots, b_{i N}\right)$ be a point in $\left(F^{\times}\right)^{N}$ such that $b_{i j}^{m_{j}}=a_{i j}$ for all $1 \leq i \leq r$ and $1 \leq j \leq N$. Recall that $I_{\bar{k}}=t^{k} I_{\overline{0}}$ for all $k \in \mathbb{Z}^{N}$, and $I_{\overline{0}}$ is contained in the ideal $M_{a_{i}}$ of $R_{\overline{0}}$ for $i=1, \ldots, r$. Therefore, the map

$$
\begin{aligned}
\psi=\psi_{b}: \mathscr{L} & \rightarrow \mathfrak{g} \oplus \cdots \oplus \mathfrak{g} \quad(r \text { copies }), \\
x \otimes f & \mapsto\left(f\left(b_{1}\right) x, \ldots, f\left(b_{r}\right) x\right)
\end{aligned}
$$

descends to a well-defined Lie algebra homomorphism

$$
\bar{\psi}: \mathscr{L} / \operatorname{ker} \phi \rightarrow \mathfrak{g} \oplus \cdots \oplus \mathfrak{g} .
$$


Theorem 4.3. The map $\bar{\psi}: \mathscr{L} / \operatorname{ker} \phi \rightarrow \mathfrak{g} \oplus \cdots \oplus \mathfrak{g}$ in (4.2) is a Lie algebra isomorphism.

Proof. Let $k \in \mathbb{Z}^{N}$, and let $\bar{\psi}_{\bar{k}}: \mathfrak{g}_{\bar{k}} \otimes\left(R_{\bar{k}} / I_{\bar{k}}\right) \rightarrow \mathfrak{g}_{\bar{k}} \oplus \cdots \oplus \mathfrak{g}_{\bar{k}}$ be the restriction of $\bar{\psi}$ to the graded component $\mathfrak{g}_{\bar{k}} \otimes\left(R_{\bar{k}} / I_{\bar{k}}\right)$ of $\mathscr{L} / \operatorname{ker} \phi$.

The map $\bar{\psi}$ is injective if each $\bar{\psi}_{\bar{k}}$ is injective. In the notation of (2.3), if

$$
u=\sum_{j=1}^{\operatorname{dim} \mathfrak{g}_{\bar{k}}} x_{\bar{k} j} \otimes\left(t^{k} f_{j}(t)+I_{\bar{k}}\right)
$$

is in the kernel of $\bar{\psi}_{\bar{k}}$ for some collection of $f_{j} \in R_{\overline{0}}$, then $b_{i}^{k} f_{j}\left(b_{i}\right)=0$ for all $i$ and $j$. Then for all $i$ and $j$, we have $f_{j}\left(b_{i}\right)=0$ and $f_{j} \in M_{a_{i}}$, where $M_{a_{i}}$ is the ideal of $R_{\overline{0}}$ generated by $\left\{t_{\ell}^{m_{\ell}}-a_{i \ell} \mid \ell=1, \ldots, N\right\}$. Hence $f_{j} \in \bigcap_{i=1}^{r} M_{a_{i}}=I_{\overline{0}}$, so $t^{k} f_{j}(t) \in t^{k} I_{\overline{0}}=I_{\bar{k}}$, and

$$
\sum_{j=1}^{\operatorname{dim} \mathfrak{g}_{\bar{k}}} x_{\bar{k} j} \otimes t^{k} f_{j}(t) \in \mathfrak{g}_{\bar{k}} \otimes I_{\bar{k}} \subseteq \operatorname{ker} \phi .
$$

Hence $u=0$ in $\mathscr{L} / \operatorname{ker} \phi$, so $\bar{\psi}_{\bar{k}}$ (and thus $\bar{\psi}$ ) is injective.

By Lemma $4.1, \operatorname{dim}\left(R_{\bar{\ell}} / I_{\bar{\ell}}\right)=\operatorname{dim}\left(R_{\overline{0}} / I_{\overline{0}}\right)$ for all $\ell \in \mathbb{Z}^{N}$. Therefore,

$$
\operatorname{dim}(\mathscr{L} / \operatorname{ker} \phi)=\sum_{\bar{\ell} \in G}\left(\operatorname{dim} \mathfrak{g}_{\bar{\ell}}\right)\left(\operatorname{dim}\left(R_{\bar{\ell}} / I_{\bar{\ell}}\right)\right)=\operatorname{dim}\left(R_{\overline{0}} / I_{\overline{0}}\right) \operatorname{dim} \mathfrak{g} .
$$

Since $F$ is algebraically closed, $R_{\overline{0}} / M_{a_{i}} \cong F$ for every $i$, so the ( $F$-vector space) dimensions satisfy

$$
\operatorname{dim}\left(R_{\overline{0}} / I_{\overline{0}}\right)=\operatorname{dim}\left(R_{\overline{0}} / M_{a_{1}} \times \cdots \times R_{\overline{0}} / M_{a_{r}}\right)=r,
$$

by Corollary 3.3. Therefore, $\bar{\psi}$ is an injective homomorphism between two Lie algebras of equal dimension, so $\bar{\psi}$ is an isomorphism.

The finite-dimensional simple modules over direct sums of copies of the Lie algebra $\mathfrak{g}$ are tensor products of finite-dimensional simple modules over $\mathfrak{g}$. (See [Bourbaki 1958, section 7, numéro 7] for instance.) We can thus conclude that the finite-dimensional simple modules for multiloop algebras are pullbacks (under $\psi$ ) of tensor products of finite-dimensional simple modules over $\mathfrak{g}$.

Fix a Cartan subalgebra $\mathfrak{h} \subset \mathfrak{g}$, a base $\Delta$ of simple roots, and weights $\lambda_{i} \in \mathfrak{h}^{*}$ for $i=1, \ldots, r$. Then we will write $V_{\lambda_{i}}\left(b_{i}\right)$ for the simple $\mathfrak{g}$-module $V_{\lambda_{i}}$ of highest weight $\lambda_{i}$, equipped with the $\mathscr{L}$-action given by

$$
(x \otimes f(t)) \cdot v=f\left(b_{i}\right) x v \quad \text { for all } x \otimes f \in \mathscr{L} \text { and } v \in V_{\lambda_{i}} .
$$


The tensor product of such a family of evaluation modules will be denoted

$$
V(\lambda, b)=V_{\lambda_{1}}\left(b_{1}\right) \otimes \cdots \otimes V_{\lambda_{r}}\left(b_{r}\right),
$$

and we will write $m\left(b_{i}\right)$ for the point $\left(b_{i 1}^{m_{1}}, \ldots, b_{i N}^{m_{N}}\right) \in\left(F^{\times}\right)^{N}$ for $i=1, \ldots, r$. We have now proved one of our main results:

Corollary 4.4. Let $V$ be a finite-dimensional simple module for the multiloop algebra $\mathscr{L}$. Then there exist $b_{1}, \ldots, b_{r} \in\left(F^{\times}\right)^{N}$ and $\lambda_{1}, \ldots, \lambda_{r}$ dominant integral weights for $\mathfrak{g}$ such that $V \cong V(\lambda, b)$, where $m\left(b_{i}\right) \neq m\left(b_{j}\right)$ whenever $i \neq j$.

Conversely, if the points $m\left(b_{i}\right) \in\left(F^{\times}\right)^{N}$ are pairwise distinct, then such a tensor product of evaluation modules is simple:

Theorem 4.5. Let $\lambda_{1}, \ldots, \lambda_{r}$ be dominant integral weights for $\mathfrak{g}$, and suppose $b_{1}, \ldots, b_{r} \in\left(F^{\times}\right)^{N}$ satisfy the property that $m\left(b_{i}\right) \neq m\left(b_{j}\right)$ whenever $i \neq j$. Then $V(\lambda, b)$ is a finite-dimensional simple $\mathscr{L}$-module.

Proof. Let $I_{\overline{0}}$ be the intersection $\bigcap_{i=1}^{r} M_{a_{i}}$ of the maximal ideals $M_{a_{i}}$ of $R_{\overline{0}}$ that correspond to the points $a_{i}=m\left(b_{i}\right)$. For any $k, \ell \in \mathbb{Z}^{N}$, we see that $t^{k-\ell} I_{\overline{0}}=I_{\overline{0}}$ if $\bar{k}=\bar{\ell}$ as elements of $G=\mathbb{Z} / m_{1} \mathbb{Z} \times \cdots \times \mathbb{Z} / m_{N} \mathbb{Z}$. Thus $t^{k} I_{\overline{0}}=t^{\ell} I_{\overline{0}}$ if $\bar{k}=\bar{\ell}$, so we can unambiguously define $I_{\bar{k}}=t^{k} I_{\overline{0}}$ for any $k \in \mathbb{Z}^{N}$.

Since $a_{1}, \ldots, a_{r}$ are pairwise distinct points in $\left(F^{\times}\right)^{N}$, the proof of Theorem 4.3 (in particular, the appeal to Corollary 3.3) shows that the map

$$
\begin{aligned}
\psi: \mathscr{L} & \rightarrow \mathfrak{g} \oplus \cdots \oplus \mathfrak{g} \quad(r \text { copies }), \\
x \otimes f(t) & \mapsto\left(f\left(b_{1}\right) x, \ldots, f\left(b_{r}\right) x\right)
\end{aligned}
$$

is surjective. Then since each $V_{\lambda_{i}}$ is a simple $\mathfrak{g}$-module, we see that the tensor product $V_{\lambda_{1}} \otimes \cdots \otimes V_{\lambda_{r}}$ is a simple module over $\mathfrak{g} \oplus \cdots \oplus \mathfrak{g}$, and the pullback $V(\lambda, b)$ is a simple $\mathscr{L}$-module.

Remark 4.6. It is not difficult to verify that if $m\left(b_{i}\right)=m\left(b_{j}\right)$ for some $i \neq j$ for which $\lambda_{i}$ and $\lambda_{j}$ are both nonzero, then $V(\lambda, b)$ is not simple. However, as we do not need this fact for the classification of simple modules, we will omit its proof.

\section{Isomorphism classes of simple modules}

By Corollary 4.4 and Theorem 4.5, the finite-dimensional simple modules of the multiloop algebra $\mathscr{L}\left(\mathfrak{g} ; \sigma_{1}, \ldots, \sigma_{N}\right)$ are precisely the tensor products

$$
V(\lambda, a)=V_{\lambda_{1}}\left(a_{1}\right) \otimes \cdots \otimes V_{\lambda_{r}}\left(a_{r}\right)
$$

for which all the $\lambda_{i} \in \mathfrak{h}^{*}$ are dominant integral, and $m\left(a_{i}\right) \neq m\left(a_{j}\right)$ whenever $i \neq j$. If $\lambda_{i}=0$ for some $i$, then $V_{\lambda_{i}}\left(a_{i}\right)$ is the trivial module, and (up to isomorphism) this term can be omitted from the tensor product (5.1). With the convention that 
empty tensor products of $\mathscr{L}$-modules are the 1-dimensional trivial module, we may assume that every $\lambda_{i}$ is a nonzero dominant integral weight in (5.1).

To proceed further, we will need a lemma about how highest weights depend on triangular decompositions.

Let $L$ be a finite-dimensional semisimple Lie algebra with Cartan subalgebra $H$ and base of simple roots $\Delta \subset H^{*}$. The group Aut $L$ of automorphisms of $L$ is (canonically) a semidirect product of the group Int $L$ of inner automorphisms and the group Out $L$ of diagram automorphisms with respect to $(H, \Delta)$ :

\section{Aut $L=\operatorname{Int} L \rtimes$ Out $L$.}

See [Jacobson 1962, IX.4] for instance. Every automorphism $\theta$ can therefore be decomposed as $\theta=\tau \circ \gamma$ with an inner part $\tau \in \operatorname{Int} L$ and outer part $\gamma \in$ Out $L$.

Lemma 5.2. Let $H$ be a Cartan subalgebra of a finite-dimensional semisimple Lie algebra $L$, and let $\Delta \subset H^{*}$ be a base of simple roots. Suppose that $V$ is a finite-dimensional simple L-module of highest weight $\lambda$ with respect to $(H, \Delta)$, and $\theta \in$ Aut $L$. Write $\theta=\tau \circ \gamma$ for some $\tau \in \operatorname{Int} L$ and $\gamma \in$ Out $L$.

Then $\Delta \circ \theta^{-1}=\left\{\alpha \circ \theta^{-1} \mid \alpha \in \Delta\right\}$ is a base of simple roots for $L$, relative to the Cartan subalgebra $\theta(H) \subset L$, and $V$ has highest weight $\lambda \circ \tau^{-1}$ with respect to $\left(\theta(H), \Delta \circ \theta^{-1}\right)$.

Proof. Any diagram automorphism with respect to $(H, \Delta)$ will preserve $H$ and $\Delta$, so $V$ has highest weight $\lambda$ with respect to $\left(\gamma(H), \Delta \circ \gamma^{-1}\right)=(H, \Delta)$. Therefore, it is enough to prove the lemma for the case where $\theta=\tau$ is an inner automorphism. Since inner automorphisms are products of automorphisms of the form $\exp (\operatorname{ad} x)$ for ad-nilpotent elements $x \in L$, we may also assume without loss of generality that $\tau=\exp (\operatorname{ad} u)$ for some ad-nilpotent element $u$.

Let $\rho: L \rightarrow$ End $V$ be the homomorphism describing the action of $L$ on $V$. Then for any $v \in V$,

$$
\tau(h) . v=(\exp (\operatorname{ad} u)(h)) \cdot v=e^{\rho(u)} \rho(h) e^{-\rho(u)} v,
$$

where $e^{\rho(u)}$ denotes the matrix exponential of the endomorphism $\rho(u)$.

The map $e^{\rho(u)}$ is invertible, so for any nonzero element

$$
w \in V_{\alpha}^{H}:=\{v \in V \mid h . v=\alpha(h) v \text { for all } h \in H\},
$$

we see that $e^{\rho(u)} w \neq 0$, and using (5.3),

$$
\tau(h) . e^{\rho(u)} w=e^{\rho(u)} \rho(h) e^{-\rho(u)} e^{\rho(u)} w=\alpha(h) e^{\rho(u)} w .
$$

That is,

$$
e^{\rho(u)} V_{\alpha}^{H} \subseteq V_{\alpha \circ \tau^{-1}}^{\tau(H)}:=\left\{v \in V \mid h . v=\alpha \circ \tau^{-1}(h) . v \text { for all } h \in \tau(H)\right\} .
$$


The reverse inclusion follows similarly by considering $\tau^{-1}=\exp (-\operatorname{ad} u)$, so

$$
e^{\rho(u)} V_{\alpha}^{H}=V_{\alpha \circ \tau^{-1}}^{\tau(H)}
$$

for all $\alpha \in H^{*}$. In the case where $V$ is the adjoint module $L$, we now see that $\alpha$ is a root relative to $H$ if and only if $\alpha \circ \tau^{-1}$ is a root relative to $\tau(H)$. It follows easily that $\Delta \circ \tau^{-1}$ is a base of simple roots for $L$, with respect to the Cartan subalgebra $\tau(H)$.

The second part also follows easily, since $V_{\lambda \circ \tau^{-1}}^{\tau(H)}=e^{\rho(u)} V_{\lambda}^{H}$ is nonzero, but $V_{\lambda \circ \tau^{-1}+\alpha \circ \tau^{-1}}^{\tau(H)}=e^{\rho(u)} V_{\lambda+\alpha}^{H}=0$ for all $\alpha \in \Delta$. That is, the highest weight of $V$ is $\lambda \circ \tau^{-1}$, relative to $\left(\tau(H), \Delta \circ \tau^{-1}\right)=\left(\theta(H), \Delta \circ \theta^{-1}\right)$.

Fix a base $\Delta$ of simple roots with respect to a Cartan subalgebra $\mathfrak{h} \subseteq \mathfrak{g}$. The next theorem gives necessary and sufficient conditions for modules of the form $V(\lambda, a)$ to be isomorphic.

Theorem 5.4. Let $\lambda=\left(\lambda_{1}, \ldots, \lambda_{r}\right)$ and $\mu=\left(\mu_{1}, \ldots, \mu_{s}\right)$ be sequences of nonzero dominant integral weights with respect to $\Delta$. Suppose that $a=\left(a_{1}, \ldots, a_{r}\right)$ and $b=\left(b_{1}, \ldots, b_{s}\right)$ are sequences of points in $\left(F^{\times}\right)^{N}$ such that $m\left(a_{i}\right) \neq m\left(a_{j}\right)$ and $m\left(b_{i}\right) \neq m\left(b_{j}\right)$ whenever $i \neq j$.

Then the finite-dimensional simple $\mathscr{L}$-modules $V(\lambda, a)$ and $V(\mu, b)$ are isomorphic if and only if $r=s$ and there is a permutation $\pi \in S_{r}$ satisfying the conditions

$$
m\left(a_{i}\right)=m\left(b_{\pi(i)}\right) \quad \text { and } \quad \lambda_{i}=\mu_{\pi(i)} \circ \gamma_{i}
$$

for $i=1, \ldots, r$, where $\gamma_{i}$ is the outer part of the automorphism $\omega_{i}: \mathfrak{g} \rightarrow \mathfrak{g}$ defined by $\omega_{i}(x)=\left(b_{\pi(i)}^{k} / a_{i}^{k}\right) x$ for all $k \in \mathbb{Z}^{N}$ and $x \in \mathfrak{g}_{\bar{k}}$.

Proof. Let $\phi_{\lambda, a}: \mathscr{L} \rightarrow$ End $V(\lambda, a)$ and $\phi_{\mu, b}: \mathscr{L} \rightarrow$ End $V(\mu, b)$ be the Lie algebra homomorphisms defining the representations $V(\lambda, a)$ and $V(\mu, b)$. By Theorem 4.3, the kernel of $\phi_{\lambda, a}$ is equal to the kernel of the evaluation map $\psi_{a}$, defined by

$$
\psi_{a}: \mathscr{L} \rightarrow \mathfrak{g} \oplus \cdots \oplus \mathfrak{g}, \quad x \otimes f \mapsto\left(f\left(a_{1}\right) x, \ldots, f\left(a_{r}\right) x\right)
$$

for all $x \otimes f \in \mathscr{L}$. Similarly, $\operatorname{ker} \phi_{\mu, b}=\operatorname{ker} \psi_{b}$.

If the $\mathscr{L}$-modules $V(\lambda, a)$ and $V(\mu, b)$ are isomorphic, then $\operatorname{ker} \phi_{\lambda, a}=\operatorname{ker} \phi_{\mu, b}$, so ker $\psi_{a}=\operatorname{ker} \psi_{b}$. But ker $\psi_{a}=\bigoplus_{\bar{k} \in G} \mathfrak{g}_{\bar{k}} \otimes I_{\bar{k}}$, where $I_{\bar{k}}=t^{k} I_{\overline{0}}$ for all $k \in \mathbb{Z}^{N}$, and

$$
I_{\overline{0}}=M_{m\left(a_{1}\right)} \cap \cdots \cap M_{m\left(a_{r}\right)},
$$

where $M_{m\left(a_{i}\right)}=\left\langle t_{1}^{m_{1}}-a_{i 1}^{m_{1}}, \ldots, t_{N}^{m_{N}}-a_{i N}^{m_{N}}\right\rangle_{R_{\overline{0}}}$ is the maximal ideal of $R_{\overline{0}}$ that corresponds to the point $m\left(a_{i}\right)=\left(a_{i 1}^{m_{1}}, \ldots, a_{i N}^{m_{N}}\right)$. Since ker $\psi_{a}=\operatorname{ker} \psi_{b}$, we see 
that (in the notation of Section 3)

$$
\begin{aligned}
\left\{m\left(a_{1}\right), \ldots, m\left(a_{r}\right)\right\} & =\mathscr{V}\left(M_{m\left(a_{1}\right)} \cap \cdots \cap M_{m\left(a_{r}\right)}\right) \\
& =\mathscr{V}\left(I_{\overline{0}}\right) \\
& =\mathscr{V}\left(M_{m\left(b_{1}\right)} \cap \cdots \cap M_{m\left(b_{s}\right)}\right)=\left\{m\left(b_{1}\right), \ldots, m\left(b_{s}\right)\right\} .
\end{aligned}
$$

Hence $r=s$, and there is a permutation $\pi \in S_{r}$ such that $m\left(a_{i}\right)=m\left(b_{\pi(i)}\right)$ for $i=1, \ldots, r$. We will write $\pi(b)=\left(b_{\pi(1)}, \ldots, b_{\pi(r)}\right)$.

Let $\mathfrak{g}=\mathfrak{n}_{-} \oplus \mathfrak{h} \oplus \mathfrak{n}_{+}$be the triangular decomposition of $\mathfrak{g}$ relative to $\Delta$. Assuming that $r=s$ and $m\left(a_{i}\right)=m\left(b_{\pi(i)}\right)$ for all $i$, view $V_{\lambda}=V_{\lambda_{1}} \otimes \cdots \otimes V_{\lambda_{r}}$ and $V_{\pi(\mu)}=$ $V_{\mu_{\pi(1)}} \otimes \cdots \otimes V_{\mu_{\pi(r)}}$ as highest weight modules of the semisimple Lie algebra $\mathfrak{g}^{\oplus r}$ relative to the triangular decomposition

$$
\mathfrak{g}^{\oplus r}=\left(\mathfrak{n}_{-}^{\oplus r}\right) \oplus\left(\mathfrak{h}^{\oplus r}\right) \oplus\left(\mathfrak{n}_{+}^{\oplus r}\right) .
$$

The highest weights of $V_{\lambda}$ and $V_{\pi(\mu)}$ are $\lambda$ and $\pi(\mu)=\left(\mu_{\pi(1)}, \ldots, \mu_{\pi(r)}\right)$, respectively, where $\lambda\left(h_{1}, \ldots, h_{r}\right)=\sum_{i} \lambda_{i}\left(h_{i}\right)$ for all $\left(h_{1}, \ldots, h_{r}\right) \in \mathfrak{h}^{\oplus r}$, and $\pi(\mu) \in$ $\left(\mathfrak{h}^{\oplus r}\right)^{*}$ is defined analogously.

We can pull back the triangular decomposition (5.5) via the isomorphism $\bar{\psi}_{a}$ : $\mathscr{L} / \operatorname{ker} \psi_{a} \rightarrow \mathfrak{g}^{\oplus r}$ defined in (4.2). Then $V(\lambda, a)$ and $V(\mu, b)$ are irreducible highest weight modules of the semisimple Lie algebra $\mathscr{L} / \operatorname{ker} \psi_{a}$ relative to the triangular decomposition

$$
\mathscr{L} / \operatorname{ker} \psi_{a}=\bar{\psi}_{a}^{-1}\left(\mathfrak{n}_{-}^{\oplus r}\right) \oplus \bar{\psi}_{a}^{-1}\left(\mathfrak{h}^{\oplus r}\right) \oplus \bar{\psi}_{a}^{-1}\left(\mathfrak{n}_{+}^{\oplus r}\right) .
$$

The $\mathscr{L}$-modules $V(\lambda, a)$ and $V(\mu, b)$ are isomorphic if and only if they have the same highest weights relative to the decomposition (5.6). Since $\bar{\psi}_{a}$ maps the decomposition (5.6) to the decomposition (5.5), the highest weight of $V(\lambda, a)$ is clearly $\lambda \circ \bar{\psi}_{a}: \bar{\psi}_{a}^{-1}\left(\mathfrak{h}^{\oplus r}\right) \rightarrow F$.

The highest weight of $V(\mu, b)$ is $v \circ \bar{\psi}_{\pi(b)}$, where $v \in\left(\bar{\psi}_{\pi(b)} \bar{\psi}_{a}^{-1}\left(\mathfrak{h}^{\oplus r}\right)\right)^{*}$ is the highest weight of $V_{\pi(\mu)}$ relative to the new triangular decomposition

$$
\mathfrak{g}^{\oplus r}=\bar{\psi}_{\pi(b)} \bar{\psi}_{a}^{-1}\left(\mathfrak{n}_{-}^{\oplus r}\right) \oplus \bar{\psi}_{\pi(b)} \bar{\psi}_{a}^{-1}\left(\mathfrak{h}^{\oplus r}\right) \oplus \bar{\psi}_{\pi(b)} \bar{\psi}_{a}^{-1}\left(\mathfrak{n}_{+}^{\oplus r}\right) .
$$

Let $\bar{\psi}_{\pi(b)} \bar{\psi}_{a}^{-1}=\tau \circ \gamma$ be a decomposition into an inner automorphism $\tau$ and a diagram automorphism $\gamma$ with respect to $\left(\mathfrak{h}^{\oplus r}, \Delta\right)$. By Lemma 5.2, we have $v=\pi(\mu) \circ \tau^{-1}$, so the two modules $V(\lambda, a)$ and $V(\mu, b)$ are isomorphic if and only if $\lambda \circ \bar{\psi}_{a}=\pi(\mu) \circ \tau^{-1} \circ \bar{\psi}_{\pi(b)}$ on $\bar{\psi}_{a}^{-1}\left(\mathfrak{h}^{\oplus r}\right)$. That is, $V(\lambda, a) \cong V(\mu, b)$ if and only if

$$
\lambda=\pi(\mu) \circ \gamma
$$

on $\mathfrak{h}^{\oplus r}$. To finish the proof, it is enough to write down an explicit formula for the automorphism $\bar{\psi}_{\pi(b)} \bar{\psi}_{a}^{-1}=\tau \circ \gamma$ of $\mathfrak{g}^{\oplus r}$. 
For each $x \in \mathfrak{g}$, let $x^{i}=(0, \ldots, x, \ldots, 0) \in \mathfrak{g}^{\oplus r}$, where $x$ is in the $i$-th position. If $k \in \mathbb{Z}^{N}$ and $x \in \mathfrak{g}_{k}$, then we see that

$$
\bar{\psi}_{a}^{-1}\left(x^{i}\right)=a_{i}^{-k} x \otimes t^{k} f_{i}(t)+\operatorname{ker} \psi_{a}
$$

in $\mathscr{L} / \operatorname{ker} \psi_{a}$, for any $f_{i}(t) \in R_{\overline{0}}$ with $f_{i}\left(a_{j}\right)=\delta_{i j}$ for all $j=1, \ldots, r$. Since $f_{i} \in$ $R_{\overline{0}}=F\left[t_{1}^{ \pm m_{1}}, \ldots, t_{N}^{ \pm m_{N}}\right]$ and $m\left(a_{j}\right)=m\left(b_{\pi(j)}\right)$ for all $j$, we see that $f_{i}\left(b_{\pi(j)}\right)=\delta_{i j}$, and

$$
\bar{\psi}_{\pi(b)} \bar{\psi}_{a}^{-1}\left(x^{i}\right)=\left(b_{\pi(i)}^{k} / a_{i}^{k}\right) x^{i} .
$$

Theorem 5.4 may also be interpreted in terms of a group action on the space of parameters $(\lambda, a)$ defining the finite-dimensional simple modules of $\mathscr{L}$. Let $G^{r}=$ $G \times \cdots \times G$ ( $r$ factors $)$, where $G$ is the finite abelian group $G=\left\langle\sigma_{1}\right\rangle \times \cdots \times\left\langle\sigma_{N}\right\rangle$ as before. Note that $G$ acts on $\left(F^{\times}\right)^{N}$ via the primitive $m_{i}$-th roots of unity $\xi_{i}$ used in the definition of $\mathscr{L}$ :

$$
\left(\sigma_{1}^{c_{1}}, \ldots, \sigma_{N}^{c_{N}}\right) .\left(d_{1}, \ldots, d_{N}\right)=\left(\xi_{1}^{c_{1}} d_{1}, \ldots, \xi_{N}^{c_{N}} d_{N}\right)
$$

for any $\left(c_{1}, \ldots, c_{N}\right) \in \mathbb{Z}^{N}$ and $\left(d_{1}, \ldots, d_{N}\right) \in\left(F^{\times}\right)^{N}$. Form the semidirect product $G^{r} \rtimes S_{r}$ by letting the symmetric group $S_{r}$ act on $G^{r}$ (on the left) by permuting the factors of $G^{r}$. That is,

$$
{ }^{\pi}\left(\rho_{1}, \ldots, \rho_{r}\right)=\left(\rho_{\pi(1)}, \ldots, \rho_{\pi(r)}\right) \quad \text { for all } \pi \in S_{r} \text { and } \rho_{i} \in G .
$$

This semidirect product acts on the space of ordered $r$-tuples of points in the torus $\left(F^{\times}\right)^{N}$ by letting $G^{r}$ act diagonally and letting $S_{r}$ permute the points:

$$
\rho \pi a=\left(\rho_{1} . a_{\pi(1)}, \ldots \rho_{r} \cdot a_{\pi(r)}\right),
$$

for all $\rho=\left(\rho_{1}, \ldots, \rho_{r}\right) \in G^{r}, \pi \in S_{r}$, and $r$-tuples $a=\left(a_{1}, \ldots, a_{r}\right)$ of points $a_{i} \in\left(F^{\times}\right)^{N}$.

The group $G^{r} \rtimes S_{r}$ also acts on the space of $r$-tuples $\lambda$ of nonzero dominant integral weights. For each $\rho=\left(\rho_{1}, \ldots, \rho_{r}\right) \in G^{r}$, write $\rho_{i}=\left(\sigma_{1}^{\rho_{i 1}}, \ldots, \sigma_{N}^{\rho_{i N}}\right)$ for some nonnegative integers $\rho_{i j}$. Let the $\rho_{i}$ act on $\mathfrak{g}$ by

$$
\rho_{i}(x)=\sigma_{1}^{\rho_{i 1}} \cdots \sigma_{N}^{\rho_{i N}} x
$$

for all $x \in \mathfrak{g}$, and on the weights $\lambda_{i}$ by

$$
\rho_{i}\left(\lambda_{i}\right)=\lambda_{i} \circ \gamma\left(\rho_{i}^{-1}\right),
$$

where $\gamma\left(\rho_{i}^{-1}\right)$ is the outer part of the automorphism $\rho_{i}^{-1}: \mathfrak{g} \rightarrow \mathfrak{g}$. Then $G^{r} \rtimes S_{r}$ acts on each $\lambda=\left(\lambda_{1}, \ldots, \lambda_{r}\right)$ by

$$
\rho \pi \lambda=\left(\lambda_{\pi(1)} \circ \gamma\left(\rho_{1}^{-1}\right), \ldots, \lambda_{\pi(r)} \circ \gamma\left(\rho_{r}^{-1}\right)\right) .
$$


Combining (5.7) and (5.8) gives an action of $G^{r} \rtimes S_{r}$ on the set of pairs $(\lambda, a)$, where $\lambda$ is an $r$-tuple of nonzero dominant integral weights $\lambda_{i}$ and $a$ is an $r$-tuple of points $a_{i} \in\left(F^{\times}\right)^{N}$. Namely, let

$$
\rho \pi(\lambda, a)=\left({ }^{\rho \pi} \lambda,{ }^{\rho \pi} a\right) .
$$

In terms of this action, the isomorphism classes of the finite-dimensional simple $\mathscr{L}$-modules are labeled by orbits of the groups $G^{r} \rtimes S_{r}$.

Corollary 5.9. Let $\lambda=\left(\lambda_{1}, \ldots, \lambda_{r}\right)$ and $\mu=\left(\mu_{1}, \ldots, \mu_{s}\right)$ be sequences of nonzero dominant integral weights with respect to $\Delta$. Suppose $a=\left(a_{1}, \ldots, a_{r}\right)$ and $b=$ $\left(b_{1}, \ldots, b_{s}\right)$ are sequences of points in $\left(F^{\times}\right)^{N}$ with $m\left(a_{i}\right) \neq m\left(a_{j}\right)$ and $m\left(b_{i}\right) \neq$ $m\left(b_{j}\right)$ whenever $i \neq j$. Then $V(\lambda, a)$ and $V(\mu, b)$ are isomorphic if and only if $r=s$ and $(\lambda, a)={ }^{\rho \pi}(\mu, b)$ for some $(\rho, \pi) \in G^{r} \rtimes S_{r}$.

Proof. Note that $m\left(a_{i}\right)=m\left(b_{\pi(i)}\right)$ if and only if the coordinates $a_{i j}$ of $a_{i}=$ $\left(a_{i 1}, \ldots, a_{i N}\right)$ differ from the coordinates $b_{\pi(i) j}$ of $b_{\pi(i)}$ by an $m_{j}$-th root of unity. Since $\xi_{j}$ is a primitive $m_{j}$-th root of unity, this happens if and only if there are integers $\rho_{i j}$ such that $a_{i j}=\xi_{j}^{\rho_{i j}} b_{\pi(i) j}$. In terms of group actions, this is precisely the existence of $\rho_{i}=\left(\sigma_{1}^{\rho_{i 1}}, \ldots, \sigma_{N}^{\rho_{i N}}\right) \in G$ with $a_{i}=\rho_{i} . b_{\pi(i)}$. In other words, $m\left(a_{i}\right)=m\left(b_{\pi(i)}\right)$ for all $i$ if and only if $a={ }^{\rho \pi} b$ for some $\rho \in G^{r}$ and $\pi \in S_{r}$.

Since $\xi_{j}^{\rho_{i j}}=a_{i j} / b_{\pi(i) j}$, we see that

$$
\rho_{i}^{-1}(x)=\sigma_{1}^{-\rho_{i 1}} \cdots \sigma_{N}^{-\rho_{i N}} x=\xi_{1}^{-\rho_{i 1} k_{1}} \cdots \xi_{N}^{-\rho_{i N} k_{N}} x=\left(b_{\pi(i)}^{k} / a_{i}^{k}\right) x
$$

for all $k \in \mathbb{Z}^{N}$ and $x \in \mathfrak{g}_{\bar{k}}$. Therefore, the automorphism $\omega_{i}$ of Theorem 5.4 is equal to $\rho_{i}^{-1}$, and $\lambda={ }^{\rho \pi} \mu$ is equivalent to the condition that $\lambda_{i}=\mu_{\pi(i)} \circ \gamma_{i}$ for every $i$.

For any diagram automorphism $\sigma_{1}$, the finite-dimensional simple modules for the twisted (single) loop algebra $\mathscr{L}\left(\mathfrak{g} ; \sigma_{1}\right)$ were classified in [Chari et al. 2008]. Recently, E. Neher, A. Savage, and P. Senesi [Senesi 2009] have reinterpreted this work in terms of finitely supported $\sigma_{1}$-equivariant maps $F^{\times} \rightarrow P_{+}$, where $P_{+}$is the set of nonzero dominant integral weights of $\mathfrak{g}$ with respect to a fixed Cartan subalgebra and base of simple roots. Theorem 5.4 and Corollary 5.9 can be used to extend this perspective to the multiloop setting.

Let $\lambda=\left(\lambda_{1}, \ldots, \lambda_{r}\right)$ and $a=\left(a_{1}, \ldots, a_{r}\right)$ be as in Theorem 5.4. Each evaluation module $V_{\lambda_{i}}\left(a_{i}\right)$ corresponds to a map

$$
\chi_{\lambda_{i}, a_{i}}:\left(F^{\times}\right)^{N} \rightarrow P_{+}, \quad x \mapsto \delta_{x, a_{i}} \lambda_{i} .
$$

The isomorphism class $[\lambda, a]$ of the tensor product $V(\lambda, a)$ can then be identified with the sum of all the characters $\chi_{\eta_{0}, c_{0}}$ for which $\left(\eta_{0}, c_{0}\right)=\left(\mu_{1}, b_{1}\right)$ for some $\mu=\left(\mu_{1}, \ldots, \mu_{r}\right)$ and $b=\left(b_{1}, \ldots, b_{r}\right)$ with $(\mu, b)$ in the $G^{r} \rtimes S_{r}$-orbit of $(\lambda, a)$. 
That is, we let

$$
\chi_{[\lambda, a]}=\sum_{g \in G} \sum_{i=1}^{r} \chi_{\lambda_{i} \circ \gamma\left(g^{-1}\right), g . a_{i}} .
$$

Thus to each isomorphism class of finite-dimensional simple $\mathscr{L}\left(\mathfrak{g} ; \sigma_{1}, \ldots, \sigma_{N}\right)$ modules, we associate a finitely supported $G$-equivariant map

$$
\chi_{[\lambda, a]}:\left(F^{\times}\right)^{N} \rightarrow P_{+} .
$$

From Corollary 5.9 and the construction of $\chi_{[\lambda, a]}$, it is easy to see that distinct isomorphism classes get sent to distinct functions.

Conversely, any finitely supported $G$-equivariant map $f:\left(F^{\times}\right)^{N} \rightarrow P_{+}$corresponds to an isomorphism class $[\lambda, a]$ of finite-dimensional simple $\mathscr{L}$-modules, as follows. By $G$-equivariance, the support supp $f$ of $f$ decomposes into a disjoint union of $G$-orbits. Choose representatives $a_{1}, \ldots, a_{r} \in\left(F^{\times}\right)^{N}$ to label each $G$ orbit in $\operatorname{supp} f$. Since the $G$-orbits are disjoint, $m\left(a_{i}\right) \neq m\left(a_{j}\right)$ whenever $i \neq j$, and by definition of $f$, the $r$-tuple $\lambda:=\left(f\left(a_{1}\right), \ldots, f\left(a_{r}\right)\right)$ consists of nonzero dominant integral weights. Then by Theorem 4.5, $V(\lambda, a)$ is a finite-dimensional simple $\mathscr{L}$-module, and by Corollary 5.9 , the isomorphism class $[f]:=[\lambda, a]$ of this module is independent of the choice of orbit representatives $a_{1}, \ldots, a_{r}$. It is now straightforward to verify that $\chi_{[f]}=f$ for all finitely supported $G$-equivariant maps $f:\left(F^{\times}\right)^{N} \rightarrow P_{+}$.

Corollary 5.10. The isomorphism classes of finite-dimensional simple $\mathscr{L}$-modules are in bijection with the finitely supported $G$-equivariant maps $\left(F^{\times}\right)^{N} \rightarrow P_{+}$.

\section{Acknowledgments}

The author thanks V. Chari, E. Neher, and P. Senesi for their careful reading and comments on earlier drafts.

\section{References}

[Allison et al. 2006] B. Allison, S. Berman, and A. Pianzola, "Iterated loop algebras", Pacific J. Math. 227:1 (2006), 1-41. MR 2007g:17022 Zbl 1146.17022

[Allison et al. 2008] B. Allison, S. Berman, J. Faulkner, and A. Pianzola, "Realization of gradedsimple algebras as loop algebras", Forum Math. 20:3 (2008), 395-432. MR 2009d:17032 Zbl 1157.17009

[Allison et al. 2009] B. Allison, S. Berman, J. Faulkner, and A. Pianzola, "Multiloop realization of extended affine Lie algebras and Lie tori”, Trans. Amer. Math. Soc. 361:9 (2009), 4807-4842. MR 2506428 Zbl 05603030

[Atiyah and Macdonald 1969] M. F. Atiyah and I. G. Macdonald, Introduction to commutative algebra, Addison-Wesley, Reading, MA, 1969. MR 39 \#4129 Zbl 0175.03601

[Batra 2004] P. Batra, "Representations of twisted multi-loop Lie algebras", J. Algebra 272:1 (2004), 404-416. MR 2004k:17011 Zbl 1077.17005 
[Bourbaki 1958] N. Bourbaki, Éléments de mathématique, XXIII, Première partie: Les structures fondamentales de l'analyse, Livre II: Algèbre, Chapitre 8: Modules et anneaux semi-simples, Actualités Sci. Ind. 1261, Hermann, Paris, 1958. MR 20 \#4576 Zbl 0102.27203

[Bourbaki 1960] N. Bourbaki, Éléments de mathématique, XXVI, Groupes et algèbres de Lie, Chapitre 1: Algèbres de Lie, Actualités Sci. Ind. 1285, Hermann, Paris, 1960. MR 24 \#A2641 Zbl 0199. 35203

[Chari 1986] V. Chari, "Integrable representations of affine Lie-algebras", Invent. Math. 85:2 (1986), 317-335. MR 88a:17034 Zbl 0603.17011

[Chari and Pressley 1986] V. Chari and A. Pressley, "New unitary representations of loop groups", Math. Ann. 275:1 (1986), 87-104. MR 88f:17029 Zbl 0603.17012

[Chari and Pressley 1987] V. Chari and A. Pressley, "A new family of irreducible, integrable modules for affine Lie algebras", Math. Ann. 277:3 (1987), 543-562. MR 88h:17022 Zbl 0608.17009

[Chari and Pressley 1988] V. Chari and A. Pressley, "Integrable representations of twisted affine Lie algebras”, J. Algebra 113:2 (1988), 438-464. MR 89h:17035 Zbl 0661.17023

[Chari et al. 2008] V. Chari, G. Fourier, and P. Senesi, "Weyl modules for the twisted loop algebras", J. Algebra 319:12 (2008), 5016-5038. MR 2009e:17018 Zbl 1151.17002

[Chari et al. 2009] V. Chari, G. Fourier, and T. Khandai, "A categorical approach to Weyl modules", preprint, version 1, 2009. arXiv 0906.2014v1

[Gille and Pianzola 2007] P. Gille and A. Pianzola, "Galois cohomology and forms of algebras over Laurent polynomial rings", Math. Ann. 338:2 (2007), 497-543. MR 2008b:20055 Zbl 1131.11070

[Jacobson 1962] N. Jacobson, Lie algebras, Pure and Applied Mathematics 10, Wiley, New YorkLondon, 1962. Republished by Dover, 1979. MR 26 \#1345 Zbl 0121.27504

[Kac 1990] V. G. Kac, Infinite-dimensional Lie algebras, 3rd ed., Cambridge University Press, 1990. MR 92k:17038 Zbl 0716.17022

[Neher 2004] E. Neher, "Lie tori", C. R. Math. Acad. Sci. Soc. R. Can. 26:3 (2004), 84-89. MR 2005d:17030 Zbl 1106.17027

[Neher et al. 2009] E. Neher, A. Savage, and P. Senesi, "Irreducible finite-dimensional representations of equivariant map algebras", preprint, version 2, 2009. arXiv 0906.5189v2

[Pal and Batra 2008] T. Pal and P. Batra, "Representations of graded multi-loop Lie algebras", preprint, version 3, 2008. arXiv 0706.0448v3

[Rao 1993] S. E. Rao, "On representations of loop algebras", Comm. Algebra 21:6 (1993), 21312153. MR 95c:17039 Zbl 0777.17019

[Rao 2001] S. E. Rao, "Classification of irreducible integrable modules for multi-loop algebras with finite-dimensional weight spaces", J. Algebra 246:1 (2001), 215-225. MR 2003c:17010 Zbl 0994.17002

[Senesi 2009] P. Senesi, "Finite-dimensional representation theory of loop algebras: A survey", preprint, version 2, 2009. To appear in Contemp. Math. arXiv 0906.0099v2

Received March 7, 2009. Revised September 17, 2009.

Michael LAU

DÉPARTEMENT DE MATHÉMATIQUES ET DE STATISTIQUE

UNIVERSITÉ LAVAL

QUÉBEC, QC G1V 0A6

CANADA

Michael.Lau@mat.ulaval.ca 